\title{
Construction of a telomerase-immortalized porcine tracheal epithelial cell model for swine-origin mycoplasma infection
}

\section{Xing Xie}

Jiangsu Academy of Agricultural Sciences

Fei Hao

Jiangsu Academy of Agricultural Sciences

Haiyan Wang

Jiangsu Academy of Agricultural Sciences

Maoda Pang

Jiangsu Academy of Agricultural Sciences

Yuan Gan

Jiangsu Academy of Agricultural Sciences

Beibei Liu

Jiangsu Academy of Agricultural Sciences

Lei Zhang

Jiangsu Academy of Agricultural Sciences

Yanna Wei

Jiangsu Academy of Agricultural Sciences

Rong Chen

Jiangsu Academy of Agricultural Sciences

Zhenzhen Zhang

Jiangsu Academy of Agricultural Sciences

Wenbin Bao

Yangzhou University

\section{Yun Bai}

Jiangsu Academy of Agricultural Sciences

\section{Guoqing Shao}

Jiangsu Academy of Agricultural Sciences

Qiyan Xiong

Jiangsu Academy of Agricultural Sciences

Zhixin Feng ( $\nabla$ fzxjaas@163.com )

Jiangsu Academy of Agricultural Sciences https://orcid.org/0000-0001-9113-4706 
Research

Keywords: porcine tracheal epithelial cells (PTECs), hTERT-PTECs, swine-origin mycoplasmas, adhesion, cell model

Posted Date: August 21st, 2020

DOI: https://doi.org/10.21203/rs.3.rs-61553/v1

License: (c) (i) This work is licensed under a Creative Commons Attribution 4.0 International License.

Read Full License

Version of Record: A version of this preprint was published at Journal of Integrative Agriculture on February 1st, 2022. See the published version at https://doi.org/10.1016/S2095-3119(21)63644-4. 


\section{Abstract}

Background: Primary porcine tracheal epithelial cells (PTECs) are an appropriate model for studying the molecular mechanism of various porcine respiratory diseases, including swine-origin mycoplasmas, which were isolated from pig respiratory tract and were mainly found on the mucosal surface surrounding swine trachea. However, the short proliferation ability of primary PTECs greatly limits their lifespan.

Results: In this study, we carefully isolated and cultured primary PTECs. Besides, we constructed immortalized PTECs by transfecting primary PTECs with the recombinant constructed plasmid pEGFPhTERT containing human telomerase reverse transcriptase (hTERT). Immortal PTECs (hTERT-PTECs) maintained both the morphological and functional characteristics of primary PTECs, as indicated by the expression of cytokeratin 18, cell-cycle analysis, proliferation assay, western blotting, telomerase activity assay, karyotype analysis and quantitative RT-PCR. hTERT-PTECs had an extended replicative lifespan, higher telomerase activity, and enhanced proliferative activity. In addition, using this cell line, the lack of transformed and grown tumors in nude mice indicated that it could be safely applied in further studies. Moreover, hTERT-PTECs were vulnerable to all swine-origin mycoplasmas and were suitable as a cell adhesion model.

Conclusions: In summary, hTERT-PTECs could be widely used as an adhesion cell model for swine-origin mycoplasmas and in the infection study of various porcine respiratory pathogens.

\section{Background}

Swine respiratory tract is colonized by various pathogens, the bacteria of which comprise four common mycoplasma species, Mycoplasma hyopneumoniae (Mhp), Mycoplasma hyorhinis (Mhr), Mycoplasma flocculare (MF) and Mycoplasma hyosynoviae (MHS). Colonization by MF is virtually asymptomatic; the other three swine-origin mycoplasmas are pathogenic. Mhr is usually present in cases of arthritis and polyserositis, after which pneumonic lesions appear. MHS can occasionally exist and colonize the lower respiratory tract [1]. However, as the primary pathogen of enzootic pneumonia and primary agent participated in the porcine respiratory disease complex, the representative pathogenic Mhp will lead to chronic respiratory diseases in pigs that are highly prevalent worldwide [2]. All swine-origin mycoplasmas that are isolated from swine respiratory tract are mainly found on the mucosal surface among swine trachea. The first step of their infection is successful adhesion to the cilia of the epithelium along with the swine respiratory tract, inducing ciliostasis as well as loss of cilia [3]. Pathogenic determinants, including adhesion to host cell and evasion from the immune response, have already been revealed for Mhp and Mhr. As it is known that the primary adhesin of Mhp is P97 and its paralogues [4], repeat regions 1 and 2 belong to the most important area for adhesion of P97. Thus, ciliostasis, clumping, and cilia loss, together with direct toxic harm to the respiratory epithelium, will lead to a decline in bacteria clearance and, potentially, secondary respiratory infections [2]. Infected pigs demonstrate decreased performance and increased mortality due to secondary infections, and enzootic pneumonia caused by Mhp is therefore 
considered to be the most costly pig disease [1]. Thus, swine-origin mycoplasmas, especially Mhp, play a crucial role in porcine respiratory diseases. To understand the molecular mechanism of swine-origin mycoplasmas infection, the construction of an appropriate in vitro cell infection model is required.

The airway epithelium has been thought to participate in the infection process of both animals and human respiratory pathogens [5]. Host respiratory epithelial cells serve as a main target of pathogens in vitro, which can initiate and increase the innate immune response of the host by secreting pro- or antiinflammatory mediators that will alter cell differentiation or chemotaxis activation of immune cells $[6,7]$. Moreover, the host innate immune response is critical to protect and defeat infection by respiratory pathogens, providing an irreplaceable role in safeguarding and protecting airways from invasive substances [6, 8]. Therefore, host respiratory cells are usually applied as infection models in pathogenesis studies of various respiratory pathogens $[5,8]$. Primary porcine tracheal epithelial cells (PTECs) have been widely applied due to their close in vitro resemblance to the airway epithelium. Previous studies have demonstrated that swine-origin mycoplasma, including Mhp and Mhr, infection cause mucosal destruction close to trachea tissue, leading to a greater appearance of lymphatic hyperplasia as well as neutrophil aggregation $[3,9]$. Moreover, pathogenicity of a PB2/HA mutant from a H9N2 influenza virus strain increased in porcine differentiated tracheal epithelial cells after culturing for three passages [10]. Additionally, primary PTECs are susceptible to the cytotoxic effects of swine influenza [11] and Streptococcus suis (S. suis) [12], suggesting the importance of obtaining large quantities and reliable primary PTECs for investigation of porcine respiratory pathogens.

Primary cells are of normal origin from epithelial cells mainly isolated from tissues. However, primary cells will experience a quick lifespan crisis and usually cannot be propagated for more than six passages $[7,13]$. Because of limited number of isolated tissues and finite proliferation activity of primary cells, motivating us to find an "immortalized" cell line with high proliferation capacity that maintains increased proliferation ability to allow replication for numerous passages $[7,14,15]$. Although several studies have reported the immortalization of porcine cells, for example, the construction of immortalized porcine intestinal epithelial cell line [16], immortalized porcine luteal cells [17] and immortal porcine bronchiolar epithelial cells [8]. Compared with bronchial epithelial cells, tracheal epithelial cells are much more easily isolated in high purity. In addition, compared to bronchus, trachea, as is known that the main latent site of swine-origin mycoplasmas and was usually considered to be the main infection site of upper respiratory tract pathogens. However, no reliable porcine tracheal epithelial cell lines have been established to date.

Here, to better understand the pathogenic mechanisms of swine-origin mycoplasma infection, an appropriate in vitro cell adhesion model was constructed. First, primary PTECs were carefully isolated from a clean piglet and cultured. Subsequently, primary cells were transfected with our previous recombinant constructed plasmid pEGFP-hTERT containing human telomerase reverse transcriptase (hTERT). Eventually, the immortalized porcine respiratory cell line hTERT-PTECs was constructed after selecting cells with stable hTERT expression. hTERT-PTECs not only maintained the morphological, functional characteristics similar to primary PTECs, but also showed an extended replication ability without changing phenotypic properties or inducing cancer-associated changes. More importantly, hTERT- 
PTECs were susceptible to swine-origin mycoplasmas, and to examine whether hTERT-PTECs could be used as a cell adhesion model, the adhesion rate was tested by the color-changing-unit assay using a representative Mhp. It was also confirmed using indirect immunofluorescence, western blotting and quantitative PCR. Thus, hTERT-PTECs could be applied as a cell adhesion model for studying swine-origin mycoplasmas and not only Mhp.

\section{Materials And Methods}

\section{Experimental animals}

A snatch-farrowed porcine-colostrum-deprived (SF-pCD) piglet (seven-week-old male, large Yorkshire) with neither signs of cardio-pulmonary disorders nor under any drug treatment or vaccinated, was purchased from Nanjing Zhoubang Bio-Tech Co. Ltd. A total of nine four-week-old female BALB/c-nu/nu mice were purchased from the Animal Experiment Center of Yangzhou University. Before sacrificing the piglet, an antibody test based on a serological analysis showed that all the following pathogens were negative, including Mhp, Mhr, MHS, MF, pseudorabies virus, classical swine fever virus, type O foot and mouth disease virus, porcine respiratory and reproductive syndrome virus (PRRSV), swine influenza virus of H1 and $\mathrm{H} 3$ subtype and porcine circovirus type 2 (PCV2).

\section{Swine-origin mycoplasma strains and culturing}

Swine-origin mycoplasma strains are those strains isolated from swine respiratory tract, including Mhp, Mhr, MF and MHS strains. Here, all mycoplasma strains were thawed from frozen stocks to subculture for three passages before use. Mhp strain J (ATCC 25934), MF strain (ATCC 27716) and MHS strain (ATCC 27095) were passaged once to establish frozen stocks from the ATCC. Mhp Strain 168L was gradually attenuated by continuous passage to the 350th passage from Mhp isolate 168, which was isolated from a piglet displaying typical features of mycoplasmal pneumonia in swine (MPS) in Gansu Province, China [18]. Thus, the Mhp 168L used herein was passage 353. Mhp Strain JS is a virulent strain that can induce typical characteristics of MPS with a lung lesion score of approximately 15, as described previously [19]. Mhp LH is a high virulent clinical strains that was isolated in our lab. Mhr HEF16 and 100928 are representative clinical strains that were isolated in our lab and that can induce polyserositis and arthritis, among which Mhr HEF16 has been stored as several stocks in the China Center for Type Culture Collection under CCTCC NO. M2017462. The two Mhp strains and Mhr strains were subcultured for 10 passages to establish frozen stocks after strain identification.

All Mhp and Mhr strains were cultured in modified Friis medium designated KM2 cell free medium containing 20\% (v/v) swine serum (produced in our lab from a clean sF-PCD piglet after irradiation sterilization) at $37^{\circ} \mathrm{C}$ in humidified incubator [19]. The MF strain was cultured in Friis medium, and the MHS strain was grown in a modified Hayflick's medium containing horse serum (Solarbio, Cat No. S9050) and arginine/mucin, as previously described [20]. Titers of all swine-origin mycoplasmas listed were 
quantified using a $50 \%$ color change unit $\left(\mathrm{CCU}_{50}\right)$ assay [21], which was modified based on the $\mathrm{CCU}$ assay [22] in culture medium and confirmed by quantitative PCR [18].

\section{Isolation and culture of primary PTECs}

Isolation of primary PTECs was performed in accordance and modified from the method for mouse respiratory epithelial cells [23] and porcine bronchial epithelial cells [8]. An overdose forelimb intravenous injection of $20 \%$ sodium pentobarbital ( $3 \mathrm{~mL} / \mathrm{kg}$; Sigma-Aldrich, Cat No. P3761) for piglet euthanasia was applied to guarantee experience of the least pain. Then, piglet epidermal tissue disinfection around thoracic skin was performed. After the rib cage was removed upon chest exposure with a cut made from the throat of the piglet to the last rib, the skin around the trachea was removed in case of throat contamination. As soon as the upper trachea was fastened with the help of a hemostatic clamp, the trachea far from the lung was immediately cut. The remaining trachea connected to the lung tissue was removed from chest and placed in a sterilized glass plate covered with ice-cold Dulbecco's Modified Eagle's Medium: Nutrient Mixture F-12 (DMEM/F12, Gibco, Cat No. 11330032) before being transferred to a biosafety cabinet.

Subsequently, isolated trachea tissues were placed in a 50-mL tube (Thermo Fisher, Cat No. 339652), inverted 20 times, and washed with ice-cold DMEM/F12 until the liquid became clear and no mucus was produced. The tracheal tissues were manually cut into approximately $1 \mathrm{~mm}^{3}$ pieces with the help of microscopic scissors before mincing the tissues digested in digestion solution in a $37^{\circ} \mathrm{C}$ water bath for 50 min. The digestion solution consisted of a total of $25 \mathrm{~mL}$ DMEM/F12 medium (Gibco), including $5 \mathrm{~mL}$ of 0.0005 M EDTA (diluted 1000 times, Invitrogen, Cat No. AM9260G), $10 \mathrm{~mL}$ of collagenase type I (5 $\mathrm{mg} / \mathrm{mL}$; Sigma-Aldrich, Cat No. SCR103) and $10 \mathrm{~mL}$ of $0.25 \%$ trypsin (Gibco, Cat No. 25200056). Digestion was stopped by adding a total of $2.5 \mathrm{~mL}$ fetal bovine serum (FBS; Gibco, Cat No.10099141) directly after separation and rounding of the cells. After gentle pipetting through 200 mesh filters, the crude cell-containing digestion suspension after filtering was collected in a $50-\mathrm{mL}$ tube for centrifugation for $10 \mathrm{~min}$ at $1500 \mathrm{rpm}$. The cells were resuspended in $40 \mathrm{~mL}$ of $10 \%$ DMEM/F12 medium and separately slowly added to two $\mathrm{T} 75$ cell flasks ( $20 \mathrm{~mL}$ per flask) before placing them into a $37^{\circ} \mathrm{C}$ cell incubator with a $5 \% \mathrm{CO}_{2}$ atmosphere for cell attachment purification. After $2.5 \mathrm{~h}$, the supernatant was carefully aspirated, followed by another centrifugation before the cell precipitate was resuspended in DMEM/F12 medium containing 10\% FBS together with $100 \mathrm{U} / \mathrm{mL}$ penicillin (Gibco, Cat No.15070063), $0.1 \mathrm{mg} / \mathrm{mL}$

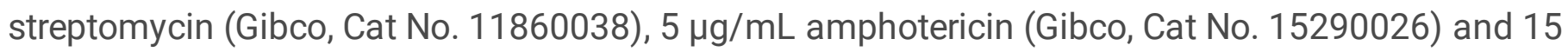
$\mu \mathrm{g} / \mathrm{mL}$ enrofloxacin (Sigma-Aldrich, Cat No. PHR1513). The cells were gently pipetted and mixed before being cultured in four 100-mm cell culture dishes biocoated with Collagen I (Corning, Cat No. 354450).

Culture medium was altered with DMEM/F12 containing $3 \%$ FBS after $18 \mathrm{~h}$ for cell attachment to inhibit the outgrowth of other cells, including smooth muscle cells and other nonepithelial cells. The PTEC cultures were supplemented with the above antibiotics and epithelial cell growth factors (LONZA, Cat No. CC-4175). Every other day, the culture medium was changed once, supplemented with an additional 20 $\mathrm{ng} / \mathrm{mL}$ hEGF (Sigma-Aldrich, Cat No. E9644) as well as $0.2 \mathrm{ng} / \mathrm{mL}$ retinoic acid (Sigma-Aldrich, Cat No. 
307-79-4). The morphological characteristics of the primary cells were assessed every three days by light microscopy (Zeiss). Primary PTECs were passaged upon reaching $85 \%$ confluence after culturing for 6 days and digested with $0.05 \%$ trypsin (Gibco, Cat No.25300062). Primary cultures were analyzed for mycoplasmas using a Mycoplasma PCR Detection Kit (GeneCopoeia, MD, Cat No. MP004-GC) to ensure that the primary PTECs were free from mycoplasma contamination.

\section{Cytokeratin 18 analysis of primary cell cultures}

To confirm our isolated primary cells were of epithelial cell origin, immunostaining with the epithelial cellorigin marker cytokeratin 18 was performed as previously described with some modifications [7, 23]. Specifically, primary PTECs of passage 2 were cultured in a 24-well cell plate before being fixed with $4 \%$ paraformaldehyde at room temperature (RT) for $15 \mathrm{~min}$. Among them, three wells of primary PTECs were cultured in $25 \%$ FBS with no epithelial cell regulation factors to guarantee that nonepithelial cells such as smooth muscle cells become the predominant cells (set as the negative control). The $0.1 \%$ Triton X-100 in PBS was added to cells for 3 min at RT, followed by blocking with 1\% bovine serum albumin (BSA, Beyotime, Cat No. ST023) in phosphate buffer saline (PBS) for $2 \mathrm{~h}$ before incubation with cytokeratin 18 primary monoclonal antibody (Abcam, Cat No. ab668) at a 1:500 dilution at $4^{\circ} \mathrm{C}$ overnight. After three times PBS washing, the cells were incubated with anti-mouse IgG H\&L (Texas Red $®$ ) (Abcam, Cat No. ab6787) at a dilution of 1:1000 for $1 \mathrm{~h}$ at $37^{\circ} \mathrm{C}$. Cell nuclei were visualized using DAPI staining solution for 3 min before observation by fluorescence microscopy (Zeiss). To evaluate the percentage of PTECs from primary cells, six microscopic fields were randomly selected in each three well in a 24-well plate at $100 \times$ magnification. The same cutoff values and manual exposure settings were used to count the cell numbers. Moreover, cytokeratin 18 on smooth muscle cells was used as a negative control and also observed.

\section{Construction of immortalized hTERT-PTECs}

After culturing for two passages, primary PTECs were seeded at a density of $2 \times 10^{5}$ cells/well into 24 -well cell plates, and the culture medium was replaced with fresh medium containing the abovementioned $3 \%$ FBS and antibiotics prior to transfection. When the primary PTECs reached approximately $85 \%$ confluence, the cells were transfected with our previously established recombinant plasmid pEGFP-hTERT [7] with Lipofectamine ${ }^{\circledR} 3000$ (Invitrogen, Cat No. L3000015). The pIRES2-EGFP vector was applied as a transfection control. Cells were observed using fluorescence microscopy (Zeiss) after stationary culture for $70 \mathrm{~h}$. Because pEGFP-hTERT contains green fluorescent protein (EGFP) and the neo gene, it could be applied as a marker to guarantee a successful transfection rate by with pEGFP-hTERT (final extracted endotoxin-free plasmid concentration should be more than $1 \mu \mathrm{g}$ ) into primary cells and conferred G418 antibiotic resistance. Selected positive cell clones were obtained in culture medium with $300 \mu \mathrm{g} / \mathrm{mL} \mathrm{G} 418$ (Gibco, Cat No. 15710072), and monoclonal cells appeared after approximately $7 \mathrm{~d}$ of selection. Reliable hTERT overexpression cell lines were constructed from several healthy as well as large colonies with G418 resistance. Positive cells were finally obtained after three rounds $\mathrm{G} 418$ antibiotic selection. The newly established cell line, hTERT-PTECs, was stored after being confirmed as mycoplasma negative. 


\section{Cell growth kinetics assessment and replicative lifespan determination}

Primary PTECs and immortalized hTERT-PTECs used in this study are primary PTECs at passage 4 and hTERT-PTECs at passage 60 unless specifically stated. Both cells were seeded at a density of $2.5 \times 10^{4}$ cells/well cultured in a 48-well plate, and medium changed once every two days. Five wells of cells were collected with trypsin, and the cell numbers were calculated every day until day 8 for cell growth kinetics analysis according to a previous study $[8,16]$.

To compare the proliferate ability between primary and immortalized cells, cell cycle analysis was performed. Both primary and immortalized cells were harvested with $0.25 \%$ trypsin without EDTA, fixed with $10 \mathrm{~mL} 75 \%$ ice-cold ethanol at $4^{\circ} \mathrm{C}$ overnight before washing twice with ice-cold PBS. The supernatant was discarded, and both cells were treated with $5 \mu \mathrm{g} / \mathrm{mL}$ propidium iodide in a volume of 0.3 $\mathrm{mL}$ (BD Biosciences, Cat No. 550825) for $30 \mathrm{~min}$ in the dark at RT after washing twice with ice-cold PBS. Cell numbers for cell cycle analysis should be no less than $1 \times 10^{6}$. Flow cytometry on a BD Accuri C6 (BD Biosciences) was applied, and the cell-cycle distribution was determined with FlowJo software $[7,16]$ as described previously. The percentages of gated cells in the cell cycle process included the G1/G0, S and G2/M phases.

\section{Western blotting analysis of hTERT}

As described previously [24], more than $5 \times 10^{6}$ primary PTECs, hTERT-PTECs and the positive control HEp2 cells (CLS Cat No.300397/p694_Hep-2) were harvested after twice PBS washing before the cells were extracted with total protein extraction kits (Vazyme, Cat No. E211). Cellular proteins were stored at $-80^{\circ} \mathrm{C}$ directly after rapid liquid nitrogen frozen for $30 \mathrm{~s}$. Approximately $20 \mu \mathrm{g}$ of each total protein was resolved by $10 \%$ SDS-PAGE before being transferred to a nitrocellulose filter membrane (Thermo Fisher, Cat No.77010). The membranes were blocked with $5 \%$ nonfat milk in Tris Buffered saline (TBS), which contains $0.5 \%$ Tween 20 (TBST) and were put in a $37^{\circ} \mathrm{C}$ incubator with gentle shaking for complete reaction for $2 \mathrm{~h}$. Then the membrane was followed by incubation with anti-hTERT (Santa Cruz Biotechnology, Cat No. sc-377511) or anti- $\beta$-actin (Bioworld, Cat No. BS6007MH) at 1:600 with 5\% nonfat milk in TBST in a $37^{\circ} \mathrm{C}$ incubator for $1.5 \mathrm{~h}$. After three times TBST washing, the membranes were incubated with secondary antibody (HRP-labeled goat anti-mouse lgG $(\mathrm{H}+\mathrm{L})$ (Boster, Cat No.BA1051) at 1:5000 with $5 \%$ nonfat milk in TBST for $1 \mathrm{~h}$. Incubation with electrochemiluminescence substrate reagents (Thermo Fisher, Cat No. 32109) was conducted after three times TBST washing, before the membrane was finally observed with a ChemiDoc XRS+ system (Bio-Rad).

\section{Telomerase activity assay}

Primary cells, hTERT-PTECs and positive control HEp-2 cells were subjected to a telomerase activity assay using a TeloTAGGG Telomerase PCR ELISAPLUS detection kit (Roche, Cat No. 11854666001), as described previously $[8,14]$. Positive control HEp-2 cells were subjected to $85^{\circ} \mathrm{C}$ for more than 10 min (named H-HEp-2, set as negative control) to heat-inactivate telomerase protein. Primary cells, positive control and our constructed immortal cells were adjusted to the same concentration. Absorbance 
determination was performed at $450 \mathrm{~nm}$ with a reference wavelength of $690 \mathrm{~nm}$. Every test was performed in five replicates. An absorbance less than 0.2 indicated that there was no telomerase activity (negative).

\section{Karyotype determination}

The chromosomes number determination in immortalized hTERT-PTECs was analyzed and modified as described previously [7, 16]. HTERT-PTECs were seeded in a T25 flask (Corning, Cat No. 354401) for approximately $24 \mathrm{~h}$ before cells being treated with colcemid (Gibco, Cat No.15212012) at $0.25 \mu \mathrm{g} / \mathrm{mL}$ (final concentration) and put in a $37^{\circ} \mathrm{C}$ incubator for $5 \mathrm{~h}$. The cells were then treated with trypsin, centrifuged at $1300 \mathrm{rpm}$ for $10 \mathrm{~min}$, suspended and incubated in a total of $5 \mathrm{~mL} 0.075 \mathrm{M} \mathrm{KCl}$ in a $15-\mathrm{mL}$ tube for $20 \mathrm{~min}$ in a $37^{\circ} \mathrm{C}$ water bath. Fresh ice-cold acetic acid/methanol $(1: 3, \mathrm{v} / \mathrm{v})$ solution was made, and the cells were fixed and subjected to blowing and careful pipetting with a dropper before centrifugation at $1300 \mathrm{rpm}$ and collection. This procedure was repeated once, and the remaining $0.3 \mathrm{~mL}$ was re-suspended, and every two to four drops of the dispersed cell suspension was smeared on every ice-cold glass slide from a height of at least more than 0.6 meters before air drying. The number of chromosomes in metaphase ( $\mathrm{n}=100$ cells) was observed microscopically after the chromosomes were stained with 4\% Giemsa solution for 15 min (Gibco, Cat No.10582013).

\section{Relative quantitative reverse transcriptase polymerase chain reaction (RT-PCR) assay}

Total RNA was extracted from primary and immortalized PTECs immediately after cell lysis using a Total RNA Extraction Kit (Omega, Cat No. R6834). With the HiScript® II QRT kit (+gDNA wiper) (Vazyme, Cat No. R223), more than $1.5 \mu \mathrm{g}$ of extracted RNA was reverse transcribed before being subjected to the HiScript ${ }^{\circledR}$ II One Step qRT-PCR SYBR $®$ Green Kit (Vazyme, Cat No. Q221) and run on an ABI 7500 Real Time PCR System. Sixteen genes were selected, including genes that participate in the cell-cycle process (sox17, sox2, ccnb1, ccnb2, ccna1 and ccna2) [25-28], response to oxidative stress (GPX1, DUOX1, DUOX2 and SOD1) $[25,29]$ and host innate immune response (IL-6, CSF2, IL-8, CXCL2, STAT3 and JNK1) [30-32]. Internal controls were $\beta$-actin and GAPDH. Primers are listed in Supplementary Table S1. The fold change in mRNA expression between primary and immortalized PTECs was analyzed using the $2^{-\triangle \Delta C T}$ method.

\section{In vivo nude mouse tumorigenicity assay}

A tumorigenicity assay was performed to assess the functional tumorigenicity of immortalized cells. Nine nude mice were randomly separated into three groups, which were inoculated with hTERT-PTECs, HEp-2 cells (positive control) and primary PTECs (negative control). The above cells were subcutaneously injected into the left flank of each of three nude mice ( 1 site/mouse at $1.5 \times 10^{6}$ cells). All nude mice were housed in a specific pathogen-free environment, and the appearance of tumors was determined once a week until two months followed by sacrifice.

\section{Soft agar analysis in vitro}


The soft agar in vitro assay was conducted to check tumorigenicity in vitro in accordance with previous studies $[8,14]$. The $0.5 \%$ agar in the bottom layer with $0.75 \mathrm{~mL}$ medium was seeded in one 24-well cell plate before being placed at $4^{\circ} \mathrm{C}$ overnight to solidify the gel. The next day, hTERT-PTECs, HEp-2 cells (positive control) and primary PTECs (negative control) were treated with trypsin and resuspended at four concentrations: $5 \times 10^{3}, 1 \times 10^{4}, 2 \times 10^{4}$ and $4 \times 10^{4}$ cells per $\mathrm{mL}$. Then, $0.5 \mathrm{~mL}$ of the cell suspension was prepared using $0.5 \%$ agar (final agar concentration: $0.33 \%$, Bactoagar, Thermo Fisher, Cat No.

DF0140010). The cells were spread over the lower solidified layer before being placed in a cell incubator. Colony observation was performed with a microscope once a week for at least six weeks. Even the appearance of a single colony suggested that the cells were able to undergo anchorage-independent growth.

\section{Susceptibility of hTERT-PTECs to four species swine-origin mycoplasmas}

Briefly, $2 \times 10^{5}$ cells/well were cultured in 24-well cell plates to perform $\mathrm{CCU}_{50}$ experiments to calculate the adhesion rate for various strains of the four mycoplasma species listed above. For the representative Mhp, to confirm whether immortal cells could be used as an adhesion model, $2 \times 10^{5}$ cells/well were additionally cultured in 24-well cell plates for the indirect immunofluorescence assay (IFA), and $5 \times 10^{5}$ cells/well were cultured in 12-well cell plates for western blotting and quantitative real-time PCR (qRTPCR).

For the IFA assay, hTERT-PTECs in a 24-well cell plate after Mhp infection were washed twice with PBS before fixation and blocking as described for the cytokeratin 18 immunostaining above method part, with the exception of the primary antibody, which was the mouse monoclonal antibody (anti-P97R1, made in our lab) at a dilution of 1:400. Cell visualization was conducted after staining the nuclei with DAPI for 2 min at RT with a fluorescence microscope (Zeiss). All Mhp-infected cells were analyzed in triplicate.

hTERT-PTECs after Mhp infection were washed twice in PBS and collected by $0.25 \%$ trypsin (without EDTA, Gibco, Cat No.15050065) digestion before western blotting analysis. The detailed protocol is exactly the same as the method described above in the hTERT protein western blotting section, with the exception of the primary antibody, which was the mouse monoclonal antibody anti-P97R1 (produced in our lab) or the loading control anti- $\beta$-actin (Bioworld, Cat No. BS6007MH) at the dilution of 1:500.

Moreover, DNA was extracted from the Mhp-infected cells in 12-well cell plates (Axygen, Cat No. AP-MNBF-VNA-250). DNA was quantified by qRT-PCR using the TaqMan system, and primers and TaqMan-BHQ probes were designed based on the conserved sequence of the P97 gene of Mhp. A standard curve was constructed using a 10-fold diluted standard plasmid, PMD-T-P97, before the QuantStudio R5 Real-Time PCR System was used to determine the DNA copies as described previously [18]. All Mhp-infected cells were run in triplicate. In addition, hTERT-PTECs seeded in 24-well plates were infected with the swineorigin mycoplasma strains isolated from four species for $12 \mathrm{~h}$ after three times PBS washing. The cells were then digested with $0.25 \%$ trypsin (without EDTA), and collected after the digestion being stopped. The concentration of each strain of swine-origin mycoplasmas before and after infection was calculated 
with the $\mathrm{CCU}_{50}$ test. Before inoculation, the DMEM/F12 cell culture medium was diluted (Mhp and Mhr strains were diluted 10 and 100 times) or resuspended (MF and MHS strains were resuspended in DMEM/F12 cell culture medium) after centrifugation for $20 \mathrm{~min}$ at $11000 \mathrm{rpm}$ to ensure that each strain of the four mycoplasma species was approximately set to $10^{7} \mathrm{CCU}_{50} / \mathrm{mL}$. Every strain of each species was assessed in five independent replicates.

\section{Statistical analysis}

Data from quantitative real-time PCR analysis and the cell-cycle test were analyzed using SPSS Statistics software v20.0 and FlowJo software v7.6. Cell numbers for the cell growth curve between primary and immortalized PTECs from day 6 to day 8 were analyzed by the multiple $t$ test, and telomerase activity between primary and immortalized PTECs were assessed via multiple comparisons of analysis of variance (ANOVA). $p<0.05$ was considered a significant difference, and $p<0.01$ was considered an extremely significant difference.

\section{Results}

\section{Isolation and features of isolated primary PTECs}

As demonstrated in Fig. 1A, whole larynx and tracheal tissue connected to the whole piglet lung tissues were removed, placed in a sterilized tray before removing the throat and for further tracheal isolation. For primary PTEC culture, after isolation of the tracheal tissues and digestion with collagenase I and $0.25 \%$ trypsin, the culture medium was changed from $10 \%$ FBS to only $3 \%$ FBS plus epithelial factors on the following day. As shown in Fig. 1B, primary PTECs of Passage 1 grew slowly, after six days, several small colonies appeared and the cell density exceeded $85 \%$. Primary PTECs were grown close to each other and demonstrated typical cuboidal phenotypic features similar to all mammary epithelial cells (Fig. 1B). The cytokeratin 18 test of primary PTECs was conducted directly after first passage primary cells were subcultured, confirming that our isolated primary cells were truly PTECs and not nonepithelial cells such as smooth muscle cells. Red staining of the primary PTECs was observed, while all the nuclei of cells, including smooth muscle cells, were stained blue with DAPI (Fig. 1C). The percentage for the purity evaluation of primary PTECs was $93.2 \pm 5.1 \%$. However, primary PTEC growth was competitively inhibited when the FBS concentration was increased to $25 \%$ without adding growth factors, and the predominant cells changed to smooth muscle cells. As shown in Fig. 1D, only a few part cell area that stained red represented primary PTECs, and the majority was all cell nuclei stained blue, indicating the negative control of cytokeratin 18 on smooth muscle cells.

\section{Construction of stable hTERT-PTECs}

Due to the reduced and short life span of primary PTECs, primary cells could not be cultured for more than 6 passages. Thus, primary cells were transfected with our previous recombinant constructed plasmid pEGFP-hTERT containing human telomerase reverse transcriptase (hTERT) to overexpress hTERT. Eventually, the immortalized porcine respiratory cell line hTERT-PTECs was constructed after 
selecting cells with stable hTERT expression that could be passaged for more than 60 passages. The EGFP encoded by the recombinant plasmid could be used to check the transfection success rate of hTERT-pEGFP by fluorescence microscopy visualization. Fig. 2A depicts the fluorescence quantity and intensity of hTERT-PTECs. In addition, hTERT-PTECs also showed cytokeratin 18 epithelial cell-origin marker immunostaining. The hTERT-PTECs maintained the features of epithelial cell similar to other mammary epithelial cells (Fig. 2B), and the purity of the hTERT-PTECs (passage 60) was $95.1 \pm 2.9 \%$.

\section{Extended replicative potential of hTERT-PTECs}

The growth curves of primary PTECs and immortalized hTERT-PTECs are depicted in Fig. 3A. All the cells demonstrated a rapid increase in cell propagation at $24 \mathrm{~h}$ to $48 \mathrm{~h}$ after subculture. The concentration of primary cells was lower than the immortalized cells from day 2 until day 4 (with no significant difference). Primary cells reached a peak before declining at $96 \mathrm{~h}$. However, hTERT-PTECs reached a maximal growth rate at $120 \mathrm{~h}$, and the cell concentration of hTERT-PTECs was significantly higher than primary PTECs from day 6 to day $8(p<0.01)$. There were no apparent differences between the morphological features of primary PTECs (Fig. 3B) and hTERT-PTECs (Fig. 3C). To confirm whether hTERT expression impacted cell proliferation ability, the distribution of the cell cycle was evaluated by flow cytometry. Under the same culture conditions, several differences were observed between hTERTPTECs (Fig. 3D) and primary PTECs (Fig. 3E) in the cell-cycle phases. The percentage of hTERT-PTECs in phase $S$ was $26.8 \%$, which was apparently higher than primary PTECs $(14.8 \%)$, while the cell percentage of hTERT-PTECs in G1 phase (61.6\%) was obviously lower than that of primary PTECs (73.9\%), suggesting that compared with primary cells, immortalized hTERT-PTECs have an increased lifespan and demonstrate enhanced proliferative potential.

\section{Increased telomerase activity of hTERT-PTECs}

As shown in Fig. 4A, hTERT protein was expressed in both immortalized cells (Lane 1) and positive control cells (Lane 2), but not in primary PTECs (Lane 3). To assess the telomerase activity of both primary PTECs and hTERT-PTECs, the telomeric replication amplification protocol (TRAP) was tested. HTERT-PTECs demonstrated apparently higher telomerase activity than primary PTECs. The optical density (OD) value of $450 \mathrm{~nm}$ for hTERT-PTECs was 0.857 , but the OD value of primary PTECs was only 0.209 ( $<0.21$ ). For the negative control heat-inactivated H-HEp-2 cells, the OD value of which was 0.151 ( $<$ 0.20 ); the OD value of the HEp-2 positive control was 0.910 (Fig. 4B).

\section{Stable and unchanged chromosome number of immortal hTERT-PTECs}

As depicted in Fig. 4C, immortalized hTERT-PTECs displayed not only a representative and typical karyotype but also a total of 38 normal diploid somatic chromosomes of pig $(2 n=38)$. Fig. 4D show the chromosomal rearrangements, which included one pair of sex chromosomes and 18 pairs of autosomes, the result of which were suitable with our experimental sF-pCD male piglets. 
As shown in Fig 5, expression levels of a total of 16 functional genes were performed. The expression levels were assessed for genes that participate in the cell-cycle process, namely, sox 17, sox2, ccna1, ccna2, ccnb1 as well as ccnb2, revealing significantly upregulated expression in immortalized hTERTPTECs compared with that in primary cells, with the only exception of the gene sox 17 , which was upregulated by only 1.3 fold. In contrast, the expression levels of genes participating in the innate immune response and response to oxidative stress remained stable, as indicated by a less than 2-fold change in relative mRNA levels.

\section{Immortalized cells with no tumorigenic properties in vitro and in vivo}

Two months later, no nude mouse inoculated with immortalized hTERT-PTECs (Fig. 6A) had grown tumors. In contrast, tumors were found in all the three nude mice inoculated with the positive control HEp2 cells (Fig. 6E). Samples collected from the nude mouse inoculation sites were evaluated using a histopathological test, the results of which confirmed the observations. Normal muscle tissue structures were visualized at the inoculation site of hTERT-PTECs (Fig. 6B and 6C), whereas pyogenic and tumor-like lesions were formed surrounding the tumor tissues. Histopathological findings were similar. A substantial area of infiltrating inflammatory cells of large quantities was visualized at the positive control HEp-2 injection site (Fig. 6F and 6G), which was not observed in mice inoculated with immortalized PTECs.

Six weeks later, not even a single colony was observed in the wells of hTERT-PTECs (Fig. 6D); however, for cells at the lowest density of $5 \times 10^{3}$ cells/well, there were still several colonies in the wells of positive control HEp-2 cells (Fig. 6H), suggesting that hTERT-PTECs were neither transformed nor had tumorigenic potential.

\section{Adhesion ability of swine-origin mycoplasmas to hTERT-PTECs}

As depicted in Fig. 7, for the IFA test, after immortalized cells were infected with different Mhp strains for $12 \mathrm{~h}$, microscopic examination showed that all strain-infected cells exhibited specific bright, spotted red immunofluorescent signals around the nucleus in different extent. In contrast, cells in the mock-infected groups did not show any specific immunofluorescent signals. The adhesion ability differed for immortalized hTERT-PTECs infected with highly or less virulent Mhp strains. Strong red fluorescent signals were visualized in cells around the nucleus infected with the highly virulent strain JS, the signals of which were obviously stronger than for the $J$ and $168 \mathrm{~L}$ strains with medium and low virulence.

Moreover, as demonstrated in Fig. 8A, P97R1 antigen of Mhp was detected in all Mhp strain-infected hTERT-PTECs. Much higher P97R1 protein expression was observed in the Mhp strain JS with higher virulence than the two other strains (Lane 2 is thicker than the other lanes). Similarly, DNA copies in all Mhp strain-infected cells reached higher numbers than $10^{5}$, and DNA copies of the highly virulent JS strain were greater than the less virulent strains (Fig. 8B), which indicated that immortalized hTERTPTECs were susceptible to various Mhp strains differing in virulence. 
$\mathrm{CCU}_{50}$ experiments were applied to calculate the adhesion rate for various strains of the four mycoplasma species, and each mycoplasma strain of every species consisted of three replicates. As shown in Fig. 8C, hTERT-PTECs were susceptible to all swine-origin mycoplasmas, and the adhesion rate of the Mhr strains was highest (10.00\% and $22.40 \%)$, MF was lowest $(0.40 \%)$, Mhp $(0.20 \%$ to $1.98 \%$ differed in strains) and MHS were moderate (2.24\%). Specifically, for Mhp, titers $\left(\mathrm{CCU}_{50} / \mathrm{mL}\right)$ of strain $\mathrm{LH}$, JS, J, and $168 \mathrm{~L}$ were $7.75,7,7.5$, and 7.5 (before infection), while titers after $12 \mathrm{~h}$ of infection were 5.5 , $5.25,5.5$ (LH); 5, 5.5, 5.25 (JS); 5.25, 5, 4.75 (J); and 5, 4.5, 4.75 (168L). Thus, the adhesion rate of highly virulent strains (LH and JS) were greater than the other two strains ( $\mathrm{J}$ and $168 \mathrm{~L})$, consistent with the western blotting and qPCR analyses. $\mathrm{CCU}_{50}$ titers of MHS and MF before and after infection were 6.75, 5.25 , and $(5,5.25,5),(2.75,3,2.75)$, respectively. For Mhr, $\mathrm{CCU}_{50}$ titers of strains HEF16 and 100928 were $6.5,6.5$ (before infection), and $(5.5 .5 .5,5.5),(5.75,5.75,6)$ (after infection). The results demonstrated that our immortalized hTERT-PTECs were susceptible to various swine-origin mycoplasmas not limited to Mhp.

\section{Discussion}

Mycoplasmas, the simplest self-replicating organisms, depend mainly on infected host cells for their nutrition, thus having an extremely parasitic dependence on their host for life $[1,33]$. They have evolved mechanisms to both invade and survive in hosts. Swine respiratory ciliary tracheal epithelium is mainly colonized by Mhp, Mhr, MHS and MF; MF is virtually asymptomatic, but the other three swine-origin mycoplasma infections can cause respiratory disease [1]. As the primary pathogen of enzootic pneumonia, Mhp can occur with and cause secondary infection with other porcine respiratory pathogens. More importantly, Mhp infections require a longer time to induce lesions and to be eliminated successfully than other pathogens [33]; thus, they play an irreplaceable role in porcine respiratory diseases and remain highly prevalent worldwide [2]. The adherence of mycoplasma, including the above four swine-origin mycoplasmas, to airway epithelial cells of the host is a precondition for their colonization and infection. After adherence to the ciliated respiratory epithelia, mycoplasma initially induces ciliostasis, with the cilia being destroyed and possibly leading to epithelial cell death. Adhesins are usually thought to be the main virulence factors of mycoplasmas, for example, the primary adhesin of Mhp is P97 and its paralogues [4], and repeat region 1 is the most important area of P97 for adhesion. Appropriate cultured cell lines are indispensable and important to evaluate the molecular and pathogenic mechanisms of various hosts. However, both pathogenic mechanism and virulence factors of swineorigin mycoplasmas and other swine respiratory pathogens are still poorly understood.

In studies of porcine respiratory pathogens, PTECs have already been depicted as a valuable in vitro cell infection model for S. suis [12], swine influenza [11] and Mhp [3]. In addition, a previous study has demonstrated that Mhp resides intracellularly within porcine epithelial cells [34], and Mhp would contribute to severe respiratory symptoms, indicating it is crucial to construct a porcine respiratory epithelial cell line such as PTECs. In this study, we provide a detailed protocol for isolating and culturing primary PTECs from a clean sF-pCD male piglet. A detailed method for the construction of telomerase- 
immortalized PTECs was provided for the first time, and evaluate whether the immortalized cell line could be used as a cell adhesion model for all swine-origin mycoplasmas not limited to Mhp.

In our previous study, primary PTECs were isolated for air-liquid interface culture by placing small tracheal tissues digested in a pronase/DNase solution at $2-8{ }^{\circ} \mathrm{C}$ overnight for 24 to $48 \mathrm{~h}$ [35]; however, in this study, to isolate primary PTECs, isolated tracheal tissues were digested with collagenase I and trypsin together for no more than $1 \mathrm{~h}$. The shorter digestion time of minced porcine tracheal tissues with microsurgical forceps as well as microsurgical scissors allowed a large quantity of extremely pure and reliable epithelial cells. Digestion in this manner with a shorter time has obvious advantages over isolation techniques applied in other species, including animals and human $[13,23,36]$, since an extended digestion time such as 16-48 $\mathrm{h}$ and weak enzymes may increase the possibility of other contaminating cells not of epithelial origin [5]. In addition, to enhance the growth of PTECs and avoid the outgrowth of other nonepithelial cells, including smooth muscle cells, DMEM/F12 medium containing epithelial cell growth factors was added, and the FBS concentration was decreased from $10 \%$ to only $3 \%$. Moreover, both retinoic acid and hEGF were added to primary PTEC cultures when the cells became adherent 18-24 $\mathrm{h}$ later. It was found that except for functions at the air-liquid interface, growth of retinoic acid and EGF may also play important roles in culture differentiation of other different species [8, 37].

Compared with primary cultures, immortalized cell lines have many advantages, especially the maintenance of relatively stable cell features and cell replication ability $[7,13,24]$. It is well known that spontaneous immortalization is a very rare event in most somatic cells. Thus, the introduction of foreign genes is usually required by transformation with a viral oncogene (such as the SV40 large T antigen) or through the telomerase reverse transcriptase method $[8,14,16,24]$, to enhance the probability of cell immortalization and of obtaining an immortal cell line $[7,13,36]$. Cell immortalization by viral oncoproteins, for example, SV40 large T antigen, mainly impact cell-cycle regulation, which is also a valuable method; however, compared with primary cells, it has shortcomings such as chromosomal abnormalities and changes in cell-cycle control [7, 26, 27]. More importantly, in contrast to their untransformed counterparts, tumorigenicity risks are also largely increased [7, 13]. Despite a few reports using a newborn porcine tracheal cell line (NpTr) to test intracellular changes [38], or an immortalized swine tracheal cell line to evaluate whether it can be cultured at the air-liquid interface [35], both cells were immortalized by introducing SV40 large T antigen, which largely increased the potential of tumorigenicity and changed the number of diploid chromosomes to polyploidy, indicating that the cells were not appropriate to study the pathogenesis of porcine respiratory pathogens, including swine-origin mycoplasmas.

Cellular senescence, a major cause of which may be shortened telomeres, as confirmed by previous research $[8,13,39]$, leading to normal somatic epithelial cells, precludes infinite proliferation. Reducing cell senescence through telomere shortening prevention is thought to be a good method to induce telomerase activity $[7,14,39]$. Thus, human telomerase reverse transcriptase (hTERT) overexpression in cells not only protects the telomere from shortening, but also initiates and enhances telomerase activation and prolongs cell replication $[8,16,24]$. With the hTERT overexpression strategy, several cells 
were successfully immortalized and obtained, such as a bovine type II alveolar epithelial cell line [24], an equine bronchial epithelial cell line [5], a porcine intestinal epithelial cell line [16], a canine bronchiolar epithelial cell line [7], a porcine luteal cell line [17] and a porcine bronchiolar epithelial cell line [8]. Tracheal epithelial cells are not only the main latent site of swine-origin mycoplasmas and was usually considered to be the main infection site of upper respiratory tract pathogens, in addition, compared with porcine bronchial epithelial cells, tracheal epithelial cells are much more easily isolated in high purity due to occlusion of the bronchial tissue peeling from the lung. However, no reliable immortalized porcine tracheal epithelial cell line without tumor risk has been constructed.

Here, stable and reliable immortal hTERT-PTECs were constructed through the transfection method with hTERT, and the morphological as well as functional features of hTERT-PTECs were evaluated. Cytokeratins, which function to retain the overall epithelial cell structural integrity, play an essential role in tissue specialization as well as cell differentiation $[7,40]$. Thus, cytokeratin expression is often thought of as the criteria for epithelial cells $[7,16]$, among which cytokeratin 18 immunostaining is usually applied to identify epithelial cells that are fully differentiated $[8,24,41]$. Here, primary cells as well as immortalized PTECs were subjected to cytokeratin 18 immunostaining. The results confirmed that the isolated cells were indeed epithelial cells, and no apparent differences were visualized through morphological feature assessment in either cell type. However, immortalized hTERT-PTECs, even those passaged for over 60 passages, still showed increased proliferation ability compared with primary PTECs, as revealed from the cell growth curves. Moreover, consistent with previous reports [7, 14, 24], the cell-cycle test through flow cytometry showed that compared with primary PTECs, the cell percentage in S phase increased significantly in immortalized PTECs, indicating that the replication lifespan of immoral cells was clearly enhanced.

As mentioned previously, ectopic hTERT expression in normal cells, which were negative for telomerase, was able to activate and enhance telomerase activity and has been widely applied to most normal cells as well as tumor cells for immortalization $[13,16,24]$. In this study, hTERT overexpression in immortal hTERT-PTEC was confirmed by western blot and TRAP analyses. Compared with primary PTECs, telomerase activity of hTERT-PTECs was relatively higher, which was only slightly lower than that of positive control, suggesting that hTERT overexpression contributed to the enhancement of telomerase activity in immortalized cells. All the results indicated that, unsurprisingly, immortal cells had a diploid karyotype and 38 modal chromosomes, in accordance with the karyotype sample isolated from a clean male sF-pCD piglet. Additionally, RT-PCR analysis of gene expression levels between primary and immortal cells compared with primary PTECs showed that all five genes participating in the cell-cycle process were strongly upregulated in immortalized cells. As revealed previously, the main reason the immortalized hTERT-PTECs exhibited enhanced replication ability was due to the higher expression levels of genes participating in cell-cycle processes crucial for immortalization [25-27, 32]. For other genes involved in the response to innate immunity and oxidative stress, hTERT-PTECs and primary PTECs all displayed a less than 2-fold change in relative mRNA levels, which is usually set as the criterion to distinguish significantly differentially gene expression levels [26, 30, 31]. Moreover, the immortalized 
hTERT-PTECs were neither transformed nor caused tumors, indicating that the immortalized cells could be applied safely in future studies.

Adhesion of swine-origin mycoplasmas to the respiratory epithelium, stimulating long-term inflammatory responses, inhibiting and regulating innate and adaptive immune responses, are considered essential steps for colonization and infection of an organism [2, 3, 18]. Among four species, Mhp, as the most representative and relatively comprehensively investigated, served herein as an example. The most common primary adhesin P97 antigen participates in cilium-binding specificity. The cilium-binding motif P97, which is located in the carboxyl-terminal R1 repeat region, contains various copies of the five amino acid motif repeats, $A A K P V(E)$, and different Mhp strains display different numbers of R1 repeats. Additionally, a previous study has shown that P97 protein can be expressed in large quantities only after infection with the ciliary epithelium of porcine respiratory tract $[3,18,33]$. Thus, the expression levels of P97R1 herein were used to determine the adhesion ability of various Mhp strains to hTERT-PTECs. Various Mhp strains of different virulence were selected to perform the IFA, western blotting and qRT-PCR assays to test the susceptibility of immortalized hTERT-PTECs to Mhp. As expected, P97R1 protein of Mhp and green fluorescent signals were detected in all Mhp strain-infected hTERT-PTECs, and DNA copies in all Mhp strain-infected hTERT-PTECs reached values higher than $10^{5}$. In addition, the adhesion ability of different Mhp strains on immortalized cells appeared to be positively correlated with the virulence of the strain, although further confirmation is needed. To verify whether the hTERT-PTECs could be used as an adhesion model for all swine-origin mycoplasmas not limited to Mhp, cells were cultured for the $\mathrm{CCU}_{50}$ test to calculate the adhesion rate for various strains of the four species before and after infection. It was found that hTERT-PTECs was susceptible to all four species of swine-origin mycoplasmas, and the adhesion rate was highest for the Mhr strains (10.00\% and $22.40 \%)$, lowest for MF $(0.40 \%)$, moderate for Mhp (0.20-1.98\%) and MHS (2.24\%). Altogether, these results demonstrated that our immortalized hTERT-PTECs were susceptible to all swine-origin mycoplasma species. Moreover, the IFA and real-time PCR test showed that immortalized cells were also susceptible to swine influenza virus (H1 and H3 subtype), PCV2, PRRSV of North American strain (data not shown), using the IFA method described in our previous study [8] and real-time PCR method. These results indicated that our immortalized hTERT-PTECs could be an appropriate cell model for various porcine respiratory pathogens not limited to the swine-origin mycoplasmas mentioned above.

In conclusion, detailed methods for isolating trachea from piglets as well as in vitro culture of primary PTECs are provided. Moreover, the immortalized cell line hTERT-PTECs was constructed, which maintains the morphology together with functional characteristics of primary PTECs, and could be used as an adhesion cell model for all swine-origin mycoplasmas not limited to Mhp. The construction of immortal hTERT-PTECs is of valuable significance as an infection cell model for pathogenesis studies of swine mycoplasmas and other porcine respiratory pathogens.

\section{Declarations}

\section{Ethics approval}


Animal experiments of piglet and nude mouse experiment were conducted in accordance with animal welfare, permitted by the Ethical Committee of Animal Experiments at Jiangsu Academy of Agricultural Sciences of China under approval number SYXK (Su) 2018-0010.

\section{Consent to participate}

Not applicable.

\section{Consent for publication}

Not applicable.

\section{Availability of data and material}

All the data we used and analyzed in the current study are available in the manuscript and can be obtained from the corresponding author on reasonable request.

\section{Competing interests}

All authors declare that they have no conflicts of interest.

\section{Funding}

This work was supported by the National Natural Science Foundation of China (31800161, 31700157, 31800160, 31900159, 31770193), Natural Science Foundation of Jiangsu Province (BK20180297, BK20170600), and the Independent Research Project Program of Jiangsu Key Laboratory for Food Quality and Safety-State Key Laboratory Cultivation Base, Ministry of Science and Technology (2019sy004).

\section{Authors' contributions}

XX: performed research, analyzed data, wrote paper and funded research. FH, HW, MP: performed research and funded research. YG, BL, LZ, YW: helped with experiment. RC, ZZ, QX: funded research and analyzed data. WB, YB, GS: analyzed data. ZF and QX conceived and designed research. All authors read and approved the manuscript.

\section{Acknowledgment}

Not applicable.

\section{Author's information}

${ }^{1}$ Key Laboratory for Veterinary Bio-Product Engineering, Ministry of Agriculture and Rural Affairs, Institute of Veterinary Medicine, Jiangsu Academy of Agricultural Sciences, Nanjing 210014, China. ${ }^{2}$ Key Laboratory of Control Technology and Standard for Agro-product Safety and Quality, Jiangsu Key 
Laboratory for Food Quality and Safety-State Key Laboratory Cultivation Base of Ministry of Science and Technology, Institute of Food Safety and Nutrition, Jiangsu Academy of Agricultural Sciences, Nanjing 210014, China. ${ }^{3}$ College of Animal Science and Technology, Yangzhou University, Yangzhou 225009, China.

\section{Abbreviations}

PTECs: porcine tracheal epithelial cells; hTERT: human telomerase reverse transcriptase; Mhp: Mycoplasma hyopneumoniae; Mhr: Mycoplasma hyorhinis; MF: Mycoplasma flocculare; MHS: Mycoplasma hyosynoviae; S. suis: Streptococcus suis; PRRSV: porcine respiratory and reproductive syndrome virus; PCV2: porcine circovirus type 2; DMEM/F12: Dulbecco's Modified Eagle's Medium: Nutrient Mixture F-12; FBS: fetal bovine serum; MPS: mycoplasmal pneumonia in swine; CCU: color change unit; RT: room temperature; PBS: phosphate buffer saline; BSA: bovine serum albumin; TBS: Tris Buffered saline; TBST: Tris Buffered saline containing 0.5\% Tween 20; RT-PCR: reverse transcriptase polymerase chain reaction; DAPI: 4',6-diamidino-2-phenylindole.

\section{References}

1. Ferrarini MG, Siqueira FM, Mucha SG, Palama TL, Jobard E, Elena-Herrmann B, AT RV, Tardy F, Schrank IS, Zaha A, Sagot MF. Insights on the virulence of swine respiratory tract mycoplasmas through genome-scale metabolic modeling. BMC Genom. 2016;17:353.

2. Maes D, Sibila M, Kuhnert P, Segales J, Haesebrouck F, Pieters M. Update on Mycoplasma hyopneumoniae infections in pigs: Knowledge gaps for improved disease control. Transbound Emerg Dis. 2018;65(Suppl 1):110-24.

3. Blanchard B, Vena MM, Cavalier A, Le Lannic J, Gouranton J, Kobisch M. Electron microscopic observation of the respiratory tract of SPF piglets inoculated with Mycoplasma hyopneumoniae. Vet Microbiol. 1992;30:329-41.

4. Hsu T, Minion FC. Identification of the cilium binding epitope of the Mycoplasma hyopneumoniae P97 adhesin. Infect Immun. 1998;66:4762-6.

5. Abraham G, Zizzadoro C, Kacza J, Ellenberger C, Abs V, Franke J, Schoon HA, Seeger J, Tesfaigzi Y, Ungemach FR. Growth and differentiation of primary and passaged equine bronchial epithelial cells under conventional and air-liquid-interface culture conditions. BMC Vet Res. 2011;7:26.

6. Mayer AK, Bartz H, Fey F, Schmidt LM, Dalpke AH. Airway epithelial cells modify immune responses by inducing an anti-inflammatory microenvironment. Eur J Immunol. 2008;38:1689-99.

7. Xie X, Pang M, Liang S, Yu L, Zhao Y, Ma K, Kalhoro DH, Lu C, Liu Y. Establishment and characterization of a telomerase-immortalized canine bronchiolar epithelial cell line. Appl Microbiol Biotechnol. 2015;99:9135-46.

8. Xie X, Gan Y, Pang M, Shao G, Zhang L, Liu B, Xu Q, Wang H, Feng Y, Yu Y, Chen R, Wu M, Zhang Z, Hua L, Xiong Q, Liu M, Feng Z. Establishment and characterization of a telomerase-immortalized 
porcine bronchial epithelial cell line. J Cell Physiol. 2018;233:9763-76.

9. Xiong Q, Wang J, Ji Y, Ni B, Zhang B, Ma Q, Wei Y, Xiao S, Feng Z, Liu M, Shao G. The functions of the variable lipoprotein family of Mycoplasma hyorhinis in adherence to host cells. Vet Microbiol. 2016; 186:82-89.

10. Yang W, Lambertz RLO, Punyadarsaniya D, Leist SR, Stech J, Schughart K, Herrler G, Wu NH, Meng F. Increased virulence of a PB2/HA mutant of an avian H9N2 influenza strain after three passages in porcine differentiated airway epithelial cells. Vet Microbiol. 2017;211:129-34.

11. Sreenivasan CC, Thomas M, Antony L, Wormstadt T, Hildreth MB, Wang, Hause B, Francis DH, Li F, Kaushik RS. Development and characterization of swine primary respiratory epithelial cells and their susceptibility to infection by four influenza virus types. Virology. 2019;528:152-63.

12. Mathieu-Denoncourt A, Letendre C, Auger JP, Segura M, Aragon V, Lacouture S, Gottschalk M. Limited Interactions between Streptococcus Suis and Haemophilus Parasuis in in vitro co-infection studies. Pathogens. 2018;7(1):7.

13. Hahn WC. Immortalization and transformation of human cells. Mol Cells. 2002;13:351-61.

14. He YL, Wu YH, He XN, Liu FJ, He XY, Zhang Y. An immortalized goat mammary epithelial cell line induced with human telomerase reverse transcriptase (hTERT) gene transfer. Theriogenology. 2009;71:1417-24.

15. Miyazawa K, Hondo T, Kanaya T, Tanaka S, Takakura I, Itani W, Rose MT, Kitazawa H, Yamaguchi T, Aso H. Characterization of newly established bovine intestinal epithelial cell line. Histochem Cell Biol. 2010;133:125-34.

16. Wang J, Hu G, Lin Z, He L, Xu L, Zhang Y. Characteristic and functional analysis of a newly established porcine small intestinal epithelial cell line. PLoS One. 2014;9:e110916.

17. Zhang L, Huang Y, Wang Z, Luo X, Zhang H, Du Q, Chang L, Zhao X, Tong D. Establishment and characterization of a telomerase immortalized porcine luteal cells. Theriogenology. 2017;94:105-13.

18. Yu Y, Liu M, Hua L, Qiu M, Zhang W, Wei Y, Gan Y, Feng Z, Shao G, Xiong Q. Fructose-1,6bisphosphate aldolase encoded by a core gene of Mycoplasma hyopneumoniae contributes to host cell adhesion. Vet Res. 2018;49:114.

19. Xiong Q, Wei Y, Feng Z, Gan Y, Liu Z, Liu M, Bai F, Shao G. Protective efficacy of a live attenuated Mycoplasma hyopneumoniae vaccine with an ISCOM-matrix adjuvant in pigs. Vet J. 2014;199:26874.

20. Kobisch M, Friis NF. Swine mycoplasmoses. Rev Sci Tech. 1996;15:1569-605.

21. Leigh SA, Evans JD, Branton SL, Collier SD. The effects of increasing sodium chloride concentration on Mycoplasma gallisepticum vaccine survival in solution. Avian Dis. 2008;52:136-8.

22. Furr PM, Taylor-Robinson D. Factors influencing the ability of different mycoplasmas to colonize the genital tract of hormone-treated female mice. Int J Exp Pathol. 1993;74:97-101.

23. Lam HC, Choi AM, Ryter SW. Isolation of mouse respiratory epithelial cells and exposure to experimental cigarette smoke at air liquid interface. JoVE. 2011;48:1-4. 
24. Su F, Liu X, Liu G, Yu Y, Wang Y, Jin Y, Hu G, Hua S, Zhang Y. Establishment and evaluation of a stable cattle type II alveolar epithelial cell line. PLoS One. 2013;8:e76036.

25. Chen X, Zhang Q, Bai J, Zhao Y, Wang X, Wang H, Jiang P. The nucleocapsid protein and nonstructural protein 10 of highly pathogenic porcine reproductive and respiratory syndrome virus enhance CD83 production via NF-kappaB and Sp1 signaling pathways. J Virol. 2017;91:e00986-17.

26. Ha Thi BM, Campolmi N, He Z, Pipparelli A, Manissolle C, Thuret JY, Piselli S, Forest F, Peoc'h M, Garraud O, Gain P, Thuret G. Microarray analysis of cell cycle gene expression in adult human corneal endothelial cells. PLoS One. 2014;9:e94349.

27. Tompkins DH, Besnard V, Lange AW, Keiser AR, Wert SE, Bruno MD, Whitsett JA. Sox2 activates cell proliferation and differentiation in the respiratory epithelium. Am J Resp Cell Mol. 2011;45:101-10.

28. Lange AW, Keiser AR, Wells JM, Zorn AM, Whitsett JA. Sox17 promotes cell cycle progression and inhibits TGF-beta/Smad3 signaling to initiate progenitor cell behavior in the respiratory epithelium. PLoS One. 2009;4:e5711.

29. Nicholson TL, Brockmeier SL, Sukumar N, Paharik AE, Lister JL, Horswill AR, Kehrli ME Jr, Loving CL, Shore SM, Deora R. The Bordetella Bps Polysaccharide is required for biofilm formation and enhances survival in the lower respiratory tract of swine. Infect Immun. 2017;85:e00261-17.

30. Lamontagne J, Mell JC, Bouchard MJ. Transcriptome-wide analysis of Hepatitis B virus-mediated changes to normal Hepatocyte gene expression. PLoS Pathog. 2016;12:e1005438.

31. Machugh DE, Taraktsoglou M, Killick KE, Nalpas NC, Browne JA, Hokamp SDEP, Gormley K, Magee E. DA. Pan-genomic analysis of bovine monocyte-derived macrophage gene expression in response to in vitro infection with Mycobacterium avium subspecies paratuberculosis. Vet Res. 2012;43:25.

32. Nalpas NC, Park SD, Magee DA, Taraktsoglou M, Browne JA, Conlon KM, Rue-Albrecht K, Killick KE, Hokamp K, Lohan AJ, Loftus BJ, Gormley E, Gordon SV, MacHugh DE. Whole-transcriptome, highthroughput RNA sequence analysis of the bovine macrophage response to Mycobacterium bovis infection in vitro. BMC Genom. 2013;14:230.

33. Pieters M, Daniels J, Rovira A. Comparison of sample types and diagnostic methods for in vivo detection of Mycoplasma hyopneumoniae during early stages of infection. Vet Microbiol. 2017;203:103-9.

34. Raymond BBA, Turnbull L, Jenkins C, Madhkoor R, Schleicher I, Uphoff CC, Whitchurch CB, Rohde M, Djordjevic SP. Mycoplasma hyopneumoniae resides intracellularly within porcine epithelial cells. Sci Rep. 2018;8:17697.

35. Wang H, He L, Liu B, Feng Y, Zhou H, Zhang Z, Wu Y, Wang J, Gan Y, Yuan T, Wu M, Xie X, Feng Z. Establishment and comparison of air-liquid interface culture systems for primary and immortalized swine tracheal epithelial cells. BMC Cell Biol. 2018;19:10.

36. Ramirez RD, Sheridan S, Girard L, Sato M, Kim Y, Pollack J, Peyton M, Zou Y, Kurie JM, Dimaio JM, Milchgrub S, Smith AL, Souza RF, Gilbey L, Zhang X, Gandia K, Vaughan MB, Wright WE, Gazdar AF, Shay JW, Minna JD. Immortalization of human bronchial epithelial cells in the absence of viral oncoproteins. Cancer res. 2004;64:9027-34. 
37. Yoon JH, Gray T, Guzman K, Koo JS, Nettesheim P. Regulation of the secretory phenotype of human airway epithelium by retinoic acid, triiodothyronine, and extracellular matrix. Am J Resp Cell Mol. 1997;16:724-31.

38. Leal Zimmer FMA, Moura H, Barr JR, Ferreira HB. Intracellular changes of a swine tracheal cell line infected with a Mycoplasma hyopneumoniae pathogenic strain. Microb Pathog. 2019;137:103717.

39. Bodnar AG, Ouellette M, Frolkis M, Holt SE, Chiu CP, Morin GB, Harley CB, Shay JW, Lichtsteiner S, Wright WE. Extension of life-span by introduction of telomerase into normal human cells. Science. 1998;279:349-52.

40. Calaf GM, Roy D. Cancer genes induced by malathion and parathion in the presence of estrogen in breast cells. Int J Mol Med. 2008;21:261-8.

41. Randell SH, Walstad L, Schwab UE, Grubb BR, Yankaskas JR. Isolation and culture of airway epithelial cells from chronically infected human lungs. In Vitro Cell Dev-An. 2001;37:480-9.

\section{Figures}



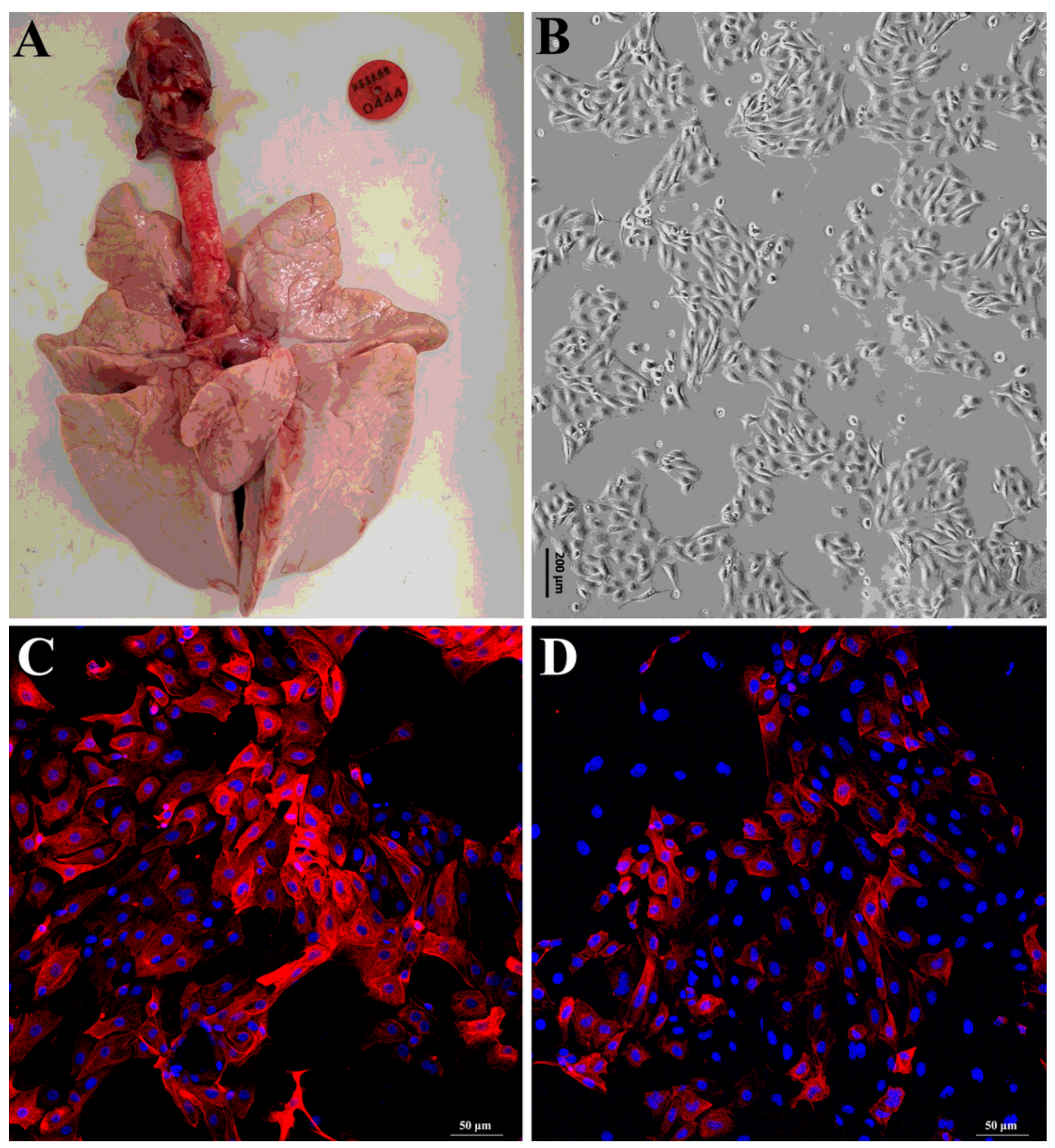

\section{Figure 1}

Isolation and culture of primary PTECs. (A): The piglet larynx and trachea connected to whole isolated lung tissue before being placed in a biosafety cabinet for further isolation. (B): Primary passage culture of PTECs depicting several colonies at $100 \times$ magnification and demonstrating a typical cuboidal phenotype similar to epithelial cells of mammals. (C): Cytokeratin immunostaining detection of primary PTECs (200 × magnification). The primary cells of the second passage were positive for cytokeratin 18, 
proving their epithelial rather than smooth muscle cell origin. (D): Immunofluorescence detection of cytokeratin 18 on smooth muscle cells (dominant cells, together with very limited primary PTECs) at 200 $\times$ magnification.

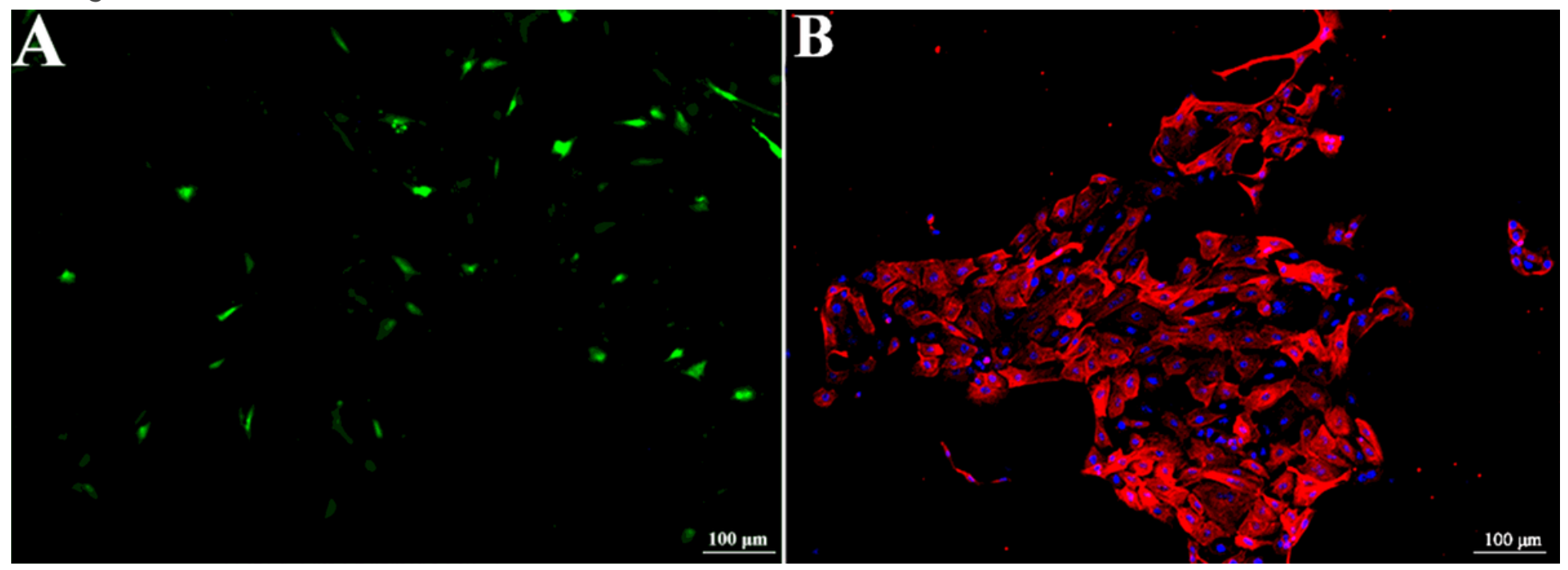

Figure 2

Fluorescence identification of immortal hTERT-PTECs. Immortalized hTERT-PTECs were transfected with the recombinant vector pEGFP-hTERT using Lipofectamine ${ }^{\circledR} 3000$. (A): Fluorescence quantity and intensity observations by fluorescence microscopy of hTERT-PTECs at passage 60 (100x magnification). (B): Cytokeratin 18 immunostaining of immortalized cells at passage 60 (100 × magnification).
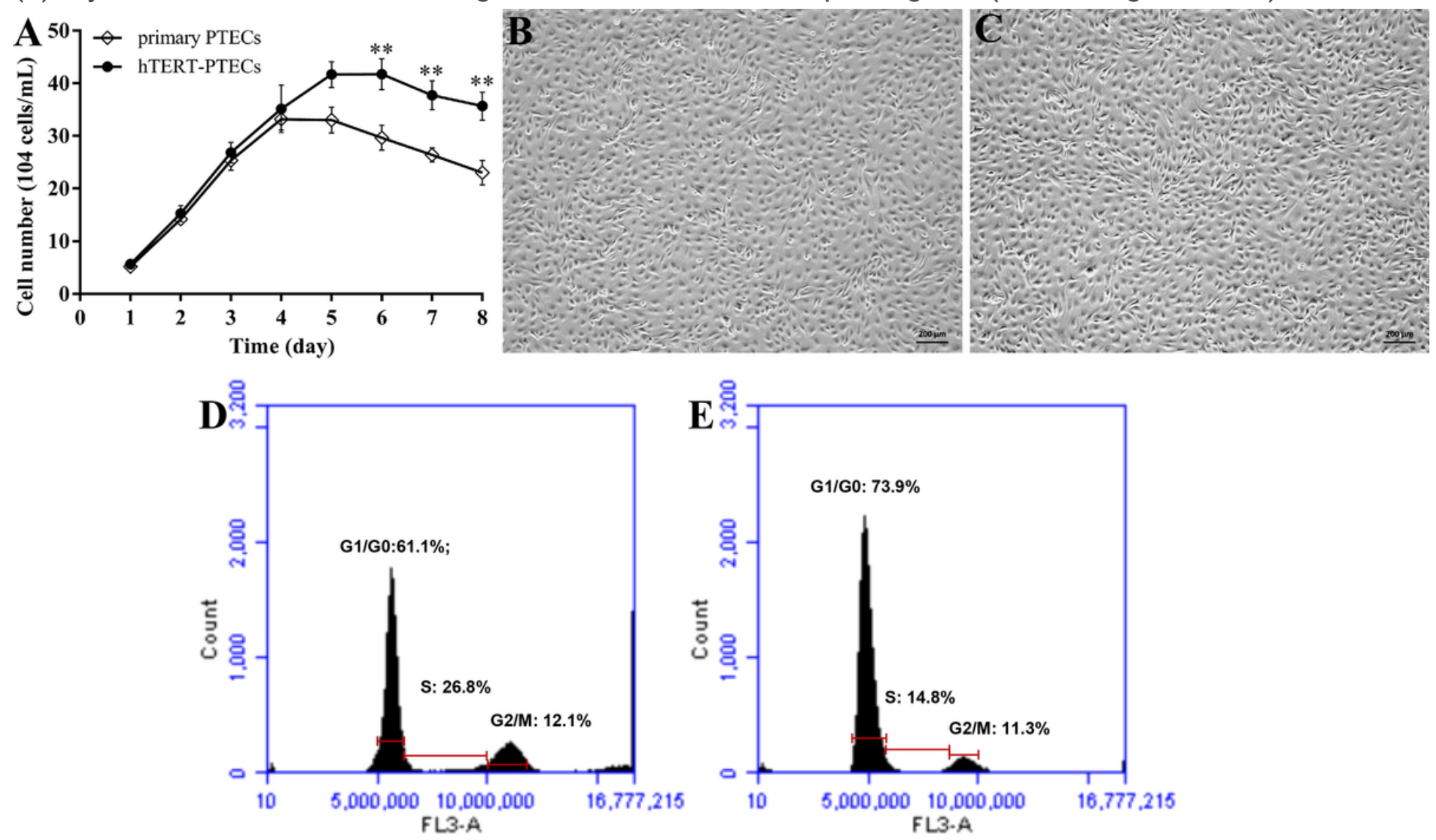
Figure 3

Proliferation capacity comparison between primary cells and immortalized hTERT-PTECs. (A): Growth curve assessment of immortalized hTERT-PTECs at passage 60 as well as control primary cells at passage 4. Data are from 5 independent experiments. ${ }^{*} p<0.01$ suggests significant differences between primary and immortal cells from day 6 to 8 . Error bars indicate standard mean variations of primary PTECs and hTERT-PTECs. (B): Morphological features of primary PTECs at passage 4. (C): Morphological features of immortal hTERT-PTECs at passage 60. (D): Cell percentage distributions for immortalized hTERT-PTECs at passage 60. (E): Cell percentage distributions of the cell cycle for primary PTECs at passage 4. Cell percentages of S phase for primary and immortal PTECs were $14.8 \%$ as well as $26.8 \%$, respectively.

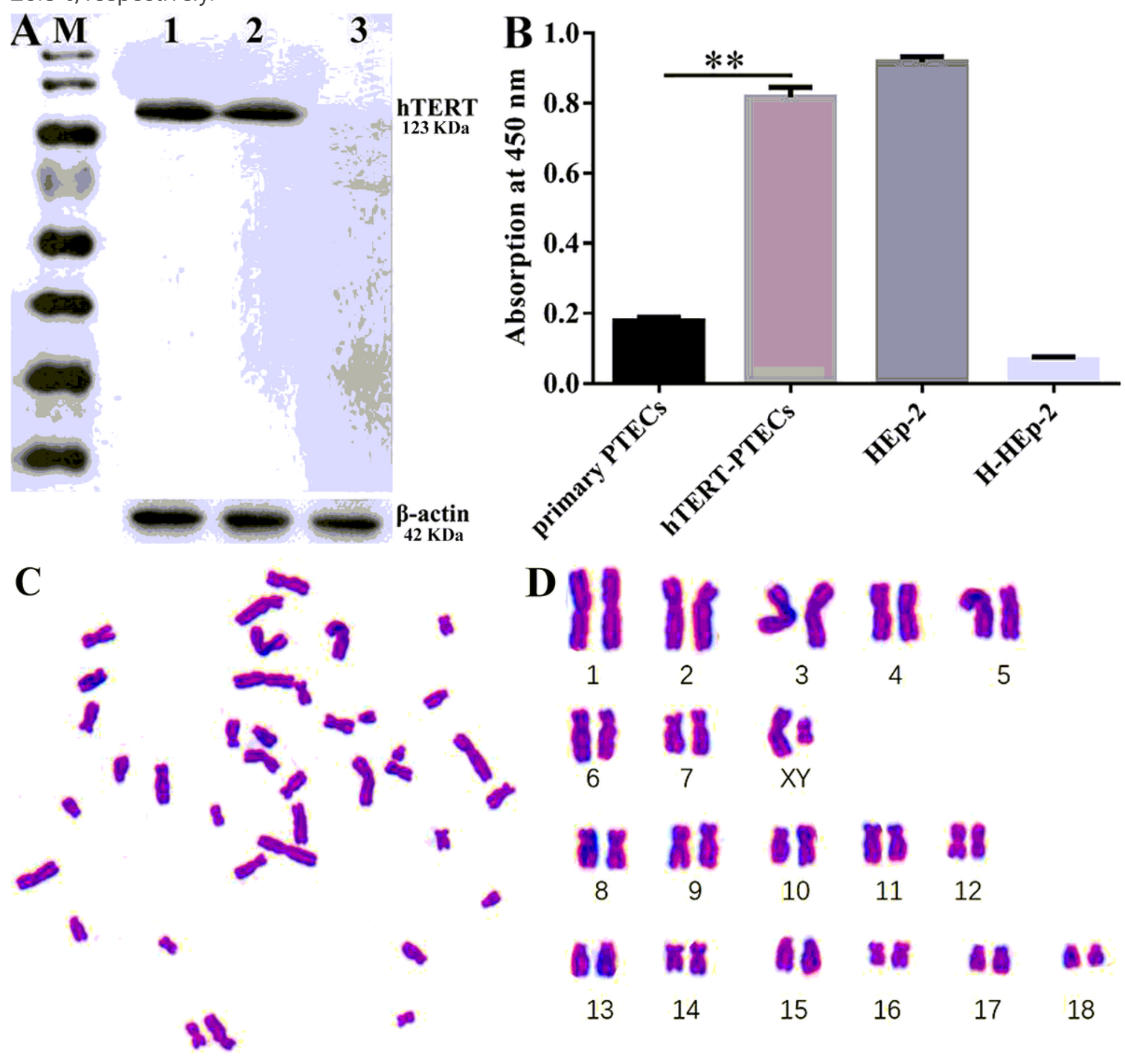


Figure 4

Characteristics and karyotype analysis of immortalized hTERT-PTECs. (A): western blotting test of hTERT. The hTERT molecular weight was $123 \mathrm{kDa}$. Lane $\mathrm{M}$ is prestained protein mass markers; Lane 1, protein expression of hTERT-PTECs at passage 60; Lane 2, protein expression of positive control HEp-2 cells; Lane 3, protein expression of negative control primary PTECs at passage 4. $\beta$-actin, an internal control, is $42 \mathrm{kDa}$. (B): Using a TeloTAGGG Telomerase PCR ELISAPLUS detection kit, telomerase activity was evaluated for immortalized hTERT-PTECs as well as primary cells. HEp-2 cells as well as heat-inactivated HEp-2 samples were positive and negative controls. Compared with primary PTECs, immortal cells demonstrated significantly higher telomerase activity $(p<0.01)$. All obtained data are the mean of five experiments for telomerase activity detection. Error bars indicate the standard mean variation of OD450 values compared with each group. (C): Karyotype test of immortalized hTERT-PTECs at passage 60. (D): Chromosomal rearrangements of immortal cells, suggesting that the cells included 38 chromosomes and confirming that they originated from a male piglet, consistent with the normal diploid karyotype.

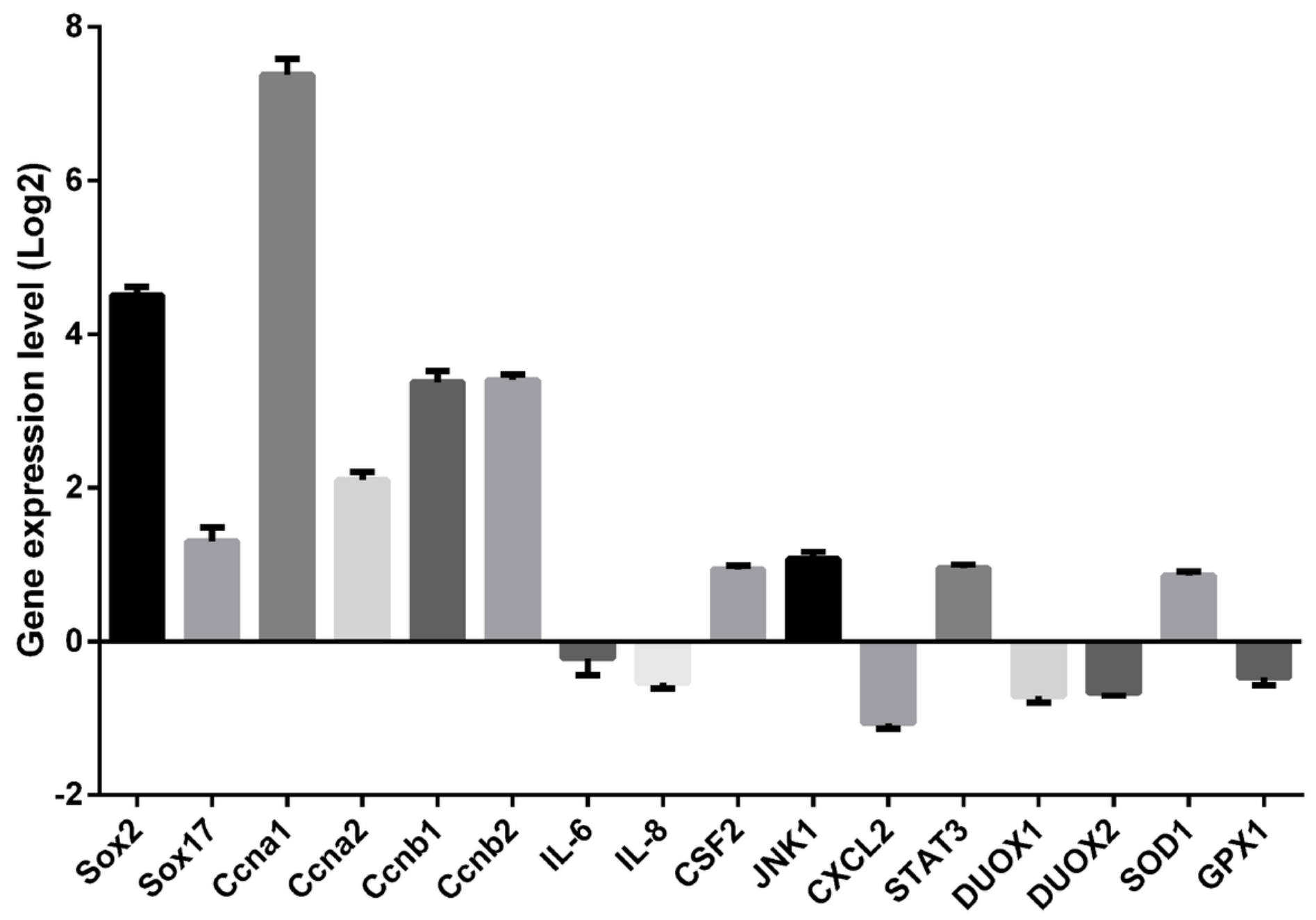

Figure 5 
Comparison of expression levels for genes participating in the cell-cycle process, response to innate immunity as well as oxidative stress in immortal and primary PTECs. For the gene expression level, primary cells were set to 1 . Error bars indicate the standard deviations obtained from three independent experiments.
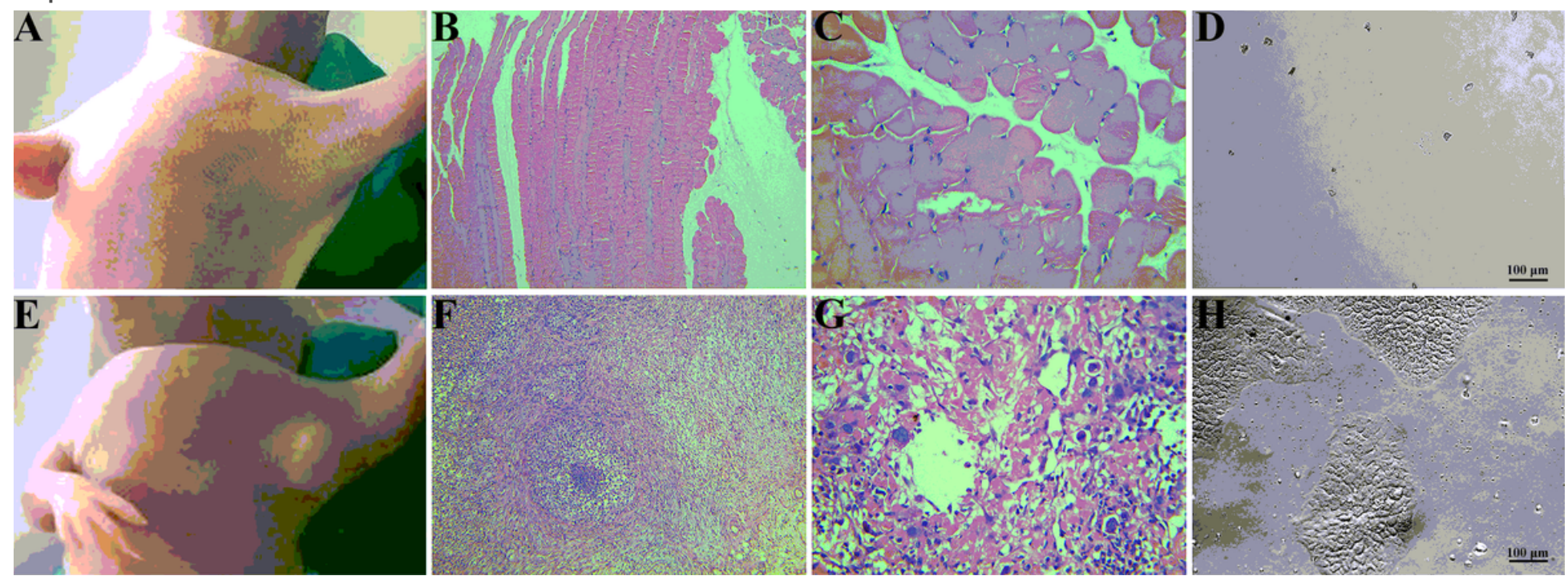

Figure 6

In vitro nude mouse tumorigenicity assay and in vivo soft agar assay of immortalized hTERT-PTECs. Primary PTECs at passage 4, hTERT-PTECs at passage 60, and positive control HEp-2 cells were subcutaneously inoculated into the left axillary region in three randomly selected nude mice. (A): No tumors were visible in all three nude mice injected with immortal hTERT-PTECs. (B): Normal tissue structure was observed from histopathological analysis below the inoculation site of the hTERT-PTECs at $100 \times$ magnification. (C): Morphologically normal tissue observation below the inoculation site of immortal cells at 400x magnification. (D): Soft agar analysis of immortalized cells at $100 \times$ magnification. (E): Three nude mice were injected with the positive control HEp-2 cells and developed tumors from the injection site two months later. $(F)$ : A large quantity of inflammatory cellular mass below the HEp-2 cell injection site was observed through histopathological analysis at $100 \times$ magnification. (G): Through histopathological examination, a dense inflammatory cellular mass was visualized under the HEp-2 cell inoculation site at $400 \times$ magnification. $(\mathrm{H})$ : Soft agar analysis of positive control HEp-2 cells seeded at a cell density of only $5 \times 103$ cells $/ \mathrm{mL}$ at $100 \times$ magnification. 


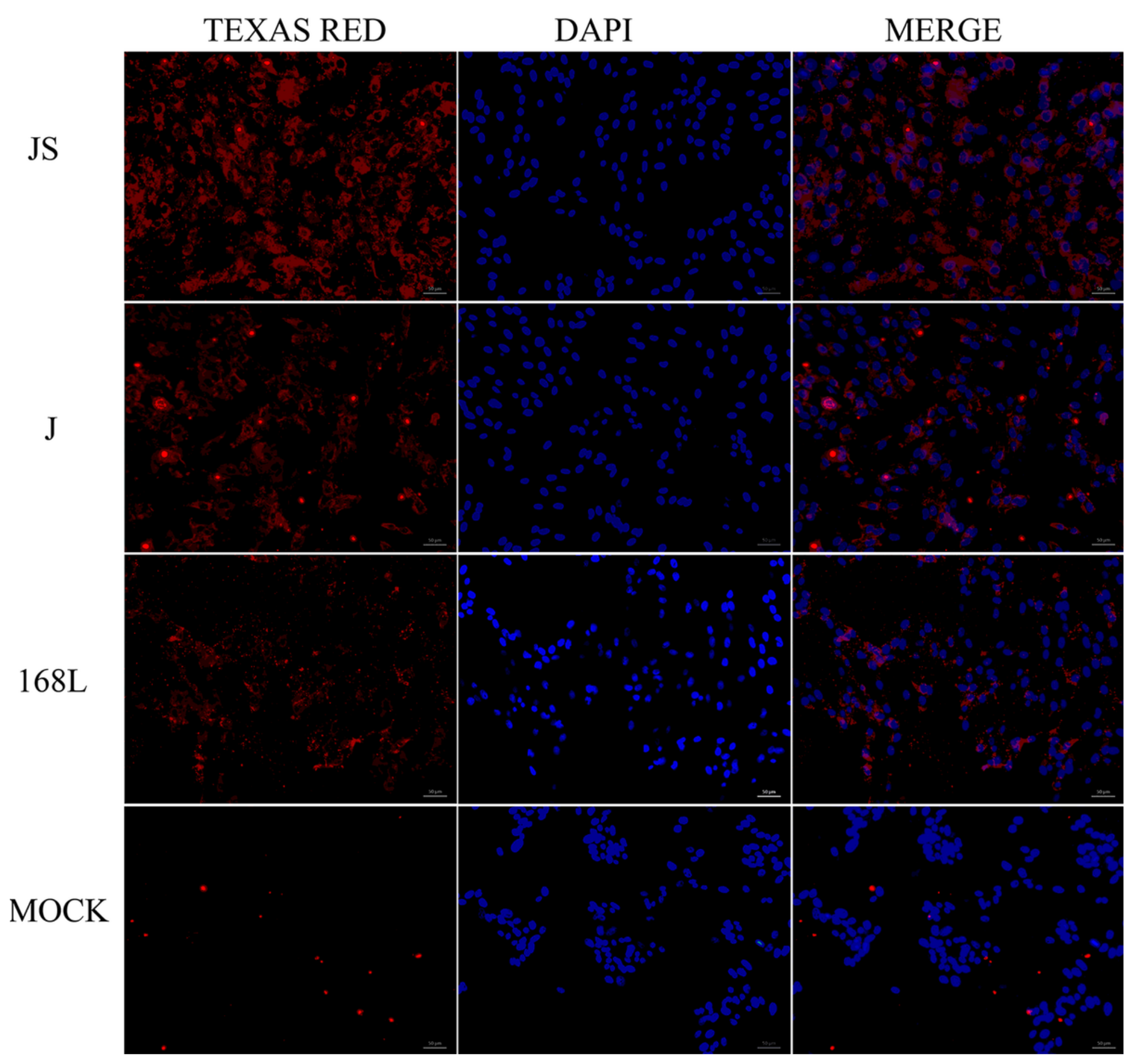

\section{Figure 7}

Immunofluorescence assay of immortalized hTERT-PTECs infected with different Mhp strains for $12 \mathrm{~h}$. Before inoculation, DMEM/F12 cell culture medium was used for dilution (Mhp strains were diluted 10 times) after centrifugation for $20 \mathrm{~min}$ at $11000 \mathrm{rpm}$ to ensure each strain of Mhp was approximately 107 CCU50/mL. Cells were stained with primary monoclonal antibody anti-P97R1 for Mhp at a 1:400 dilution and secondary antibody anti-mouse IgG H\&L (Texas Red $\circledast$ ) at a 1:1000 dilution. Texsa red, antibody reactivity to different Mhp strains observed with Texas red-conjugated anti-mouse IgG antibody (red); DAPI, 4',6-diamidino-2-phenylindole, nuclei of all cells were stained by DAPI reagent (blue); the merge of 
the images is demonstrated in the column labeled "Merge". Captured images were observed at 200x magnification. The white bar represents the scale: $50 \mu \mathrm{m}$.
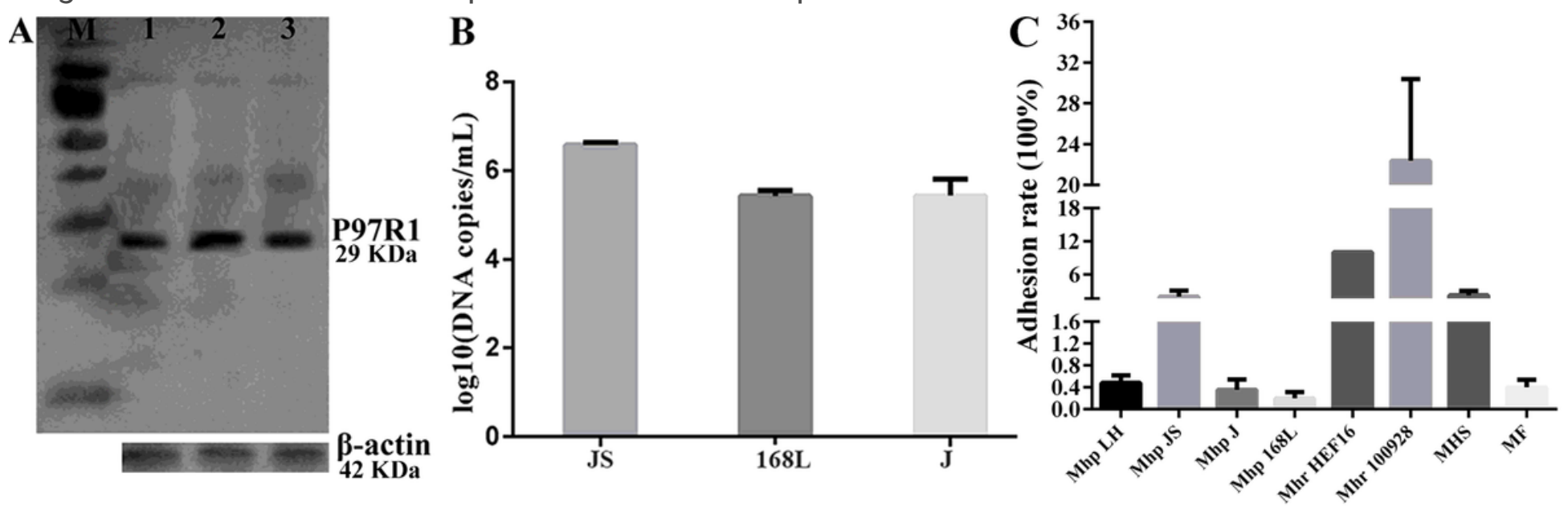

Figure 8

Western blotting and qRT-PCR assays of immortalized hTERT-PTECs infected with different Mhp strains, and CCU50 assay of immortalized cells infected with four swine-origin mycoplasmas-Mhp, Mhr, MF, and MHS-for $12 \mathrm{~h}$. Before inoculation, DMEM/F12 cell culture medium was used for dilution (Mhp and Mhr strains were diluted 10 and 100 times) or resuspension (MF and MHS strains were resuspended) after centrifugation for $20 \mathrm{~min}$ at $11000 \mathrm{rpm}$ to ensure each of the four mycoplasma species were approximately $107 \mathrm{CCU} 50 / \mathrm{mL}$. (A): P97R1 western blot analysis. The molecular weight of P97R1 was 29 kDa. Lane M, prestained protein mass markers; Lane 1, Mhp strain J; Lane 2, Mhp strain JS; Lane 3, Mhp strain 168L. $\beta$-actin, internal control, molecular weight was $42 \mathrm{kDa}$. (B): log10 DNA copy number per mL of the Mhp strain-infected cell distribution against P97 was quantified using quantitative real-time PCR. Data were obtained as the mean of five independent experiments for DNA copy determination. (C): The adhesion rate of various strains of four swine-origin mycoplasmas. Adhesion rate $=$ (CCU50 titer of each swine-origin mycoplasma strain infected for $12 \mathrm{~h} / \mathrm{CCU} 50$ titer of each swine-origin mycoplasma strain before infection) $\times 100$. Data are presented as the mean \pm SD of at least five independent experiments.

\section{Supplementary Files}

This is a list of supplementary files associated with this preprint. Click to download.

- TableS1.docx 